\title{
Verzeichnis archivalischer Quellen
}

\section{Baltimore, The Johns Hopkins University (JHU), Special Collections}

- JHU Baltimore, Special Collections, Daniel Coit Gilman Papers, 1841-1907, Series 1: Correspondence, Folders $1.2133 \quad 50$

- JHU Baltimore, Special Collections, Paul Haupt Papers, 1877-1903, MS 9047

\section{Berlin, Archiv der Berlin-Brandenburgischen Akademie der Wissenschaften} (ABBAW)

- ABBAW Berlin, NL Meyer, E, Nr. 690 (Haupt an Meyer, 13.4.1908) 56

\section{Berlin, Geheimes Staatsarchiv Preußischer Kulturbesitz (GSTAPK)}

- GSTAPK Berlin VI.HA NL Friedrich Theodor Althoff Nr. 755 (Haupt an Althoff, 27.7.1885) 50, 51

- GSTAPK Berlin, VI.HA NL Friedrich Theodor Althoff Nr. 755 (Haupt an Althoff, 1.8.1886) 60, 61

- GSTAPK Berlin, VI.HA NL Friedrich Theodor Althoff Nr. 817 (Lagarde an Althoff, 19.1.1887) 51

\section{Freiburg, Universitätsbibliothek (UB)}

- UB Freiburg Nachlass Schemann NL 12/1999 46, 53, 55, 58, 63

\section{Göttingen, Niedersächsische Staats- und Universitätsbibliothek Göttingen}

(SUB)

- SUB Göttingen, Signatur: Cod. Ms. Lagarde 150 : 170 (Brugsch) 72

- SUB Göttingen, Signatur: Cod. Ms. Lagarde 150 : 321 (Erman) 76

- SUB Göttingen, Signatur: Cod. Ms. Lagarde 150 : 321 (Erman, 16.4.1882) 93

- SUB Göttingen, Signatur: Cod. Ms. Lagarde 150 : 438 (Lagarde an von Goßler) 79

- SUB Göttingen, Signatur: Cod. Ms. Lagarde $150: 493$ (Lagarde/Haupt) 46, 49

- SUB Göttingen, Signatur: Cod. Ms. Lagarde 150 : 493 (Haupt an Lagarde, 23.7.1879) 49

- SUB Göttingen, Signatur: Cod. Ms. Lagarde 150 : 493 (Gutachten Lagardes zum Habilitationsverfahren Haupts, Dezember 1880) 48

- SUB Göttingen, Signatur: Cod. Ms. Lagarde 150 : 493 (Haupt an Lagarde, 29.8.1886) 60, 61,62

- SUB Göttingen, Signatur: Cod. Ms. Lagarde 150 : 493 (Haupt an Lagarde, 28.12.1886) 54

- SUB Göttingen, Signatur: Cod. Ms. Lagarde $150: 493$ (Haupt an Lagarde, 18.10.1887) 53

- SUB Göttingen, Signatur: Cod. Ms. Lagarde $150: 493$ (Haupt an Lagarde, 25.1.1890) 53

- SUB Göttingen, Signatur: Cod. Ms. Lagarde 150: 624 (Lagarde an Kessler, 30.3.1889) 88

- SUB Göttingen, Signatur: Cod. Ms. Lagarde 150 : 967 (Lagarde/Renan) 174

- SUB Göttingen, Signatur: Cod. Ms. Lagarde 150:1050 (Lagarde/Schemann) 46

- SUB Göttingen, Signatur: Cod. Ms. Lagarde 150 : 1160 (Steindorff, 24.12.1884) 94 
- SUB Göttingen, Signatur: Cod. Ms. Lagarde 150 : 1160 (Steindorff, 26.12.1884) 95

- SUB Göttingen, Signatur: Cod. Ms. Lagarde 150 : 1160 (Steindorff, 4.8.1886) 94

- SUB Göttingen, Signatur: Cod. Ms. Lagarde 150 : 1160 (Steindorff, 31.12.1886 und vom 4.8. 1886) 95

- SUB Göttingen, Signatur: Cod. Ms. Lagarde $150: 1160$ / Beil. (Steindorff) 39

- SUB Göttingen, Signatur: Cod. Ms. Lagarde 150 : Beil. 1 (Indizes) 39

- SUB Göttingen, Signatur: Cod. Ms. Lagarde 150 : Beil. 2 (Korrespondentenkartei) 39

- SUB Göttingen, Signatur: Cod. Ms. Lagarde $150: 1195$ (Teza an Lagarde, 25.3.1882) 41

- SUB Göttingen, Signatur: Cod. Ms. Lagarde 168 : 161 (Haupt an Anna Lagarde, 4.3.1893) 52

- SUB Göttingen, Signatur: Cod. Ms. Lagarde 168 : 161 (Haupt an Anna Lagarde, 10.12.1893) 45

- SUB Göttingen, Signatur: Cod. Ms. Lagarde 187 : 1 (letzter Wille) 36, 37

\section{Göttingen, Universitätsarchiv}

- Universitätsarchiv Göttingen, Personalakte Nr. 4 V c 80 (Vorsatzblatt: Dr. phil. Maximilian Adolph Uhlemann, †26. Juli 1862) 70

\section{Leipzig, Ägyptisches Museum - Georg Steindorff, Archiv, NI. Georg Steindorff, Korrespondenz}

- ÄMULA, Luschan-Steindorff, 4.10.1923 100

- ÄMULA, Schweinfurth-Steindorff, 22.3.1924 103

- ÄMULA, Hesse-Steindorff, 7.6.1929 103

- ÄMULA, Steindorff-Berve, 27.11.1934 104

- ÄMULA, Keimer-Steindorff, 22.10.1944 106

\section{München, Bayerische Staatsbibliothek (BSB)}

- BSB München, Ana 335 (Haupt an Hommel, 4.2.1880) 48, 49

- BSB München, Ana 335 (Haupt an Hommel, 16.3.1880) 49

\section{Washington, Smithsonian Institution (SI) Archives}

- SI Archives Washington D.C., RU 192, Box 70, Folder 2066 61, 62

- SI Archives Washington D.C., RU 201, Box 9, Folder 14 61, 62 